\title{
Latest research on long-range Brillouin distributed sensing
}

\author{
Alayn Loayssa, Javier Urricelqui, Haritz Iribas, Jon Mariñelarena, and Juan José Mompó \\ Institute of Smart Cities, Universidad Pública de Navarra, 31006 Pamplona, Spain
}

\begin{abstract}
We review the latest developments in long-range Brillouin optical time-domain analysis sensors. The factors that impair the performance of these sensors, particularly in terms of their distance range, are discussed together with the latest methods to overcome them. We focus on our recent contributions based on the application of the probe dithering method, which is based on introducing a wavelength modulation to the probe wave. This technique is shown to effectively compensate nonlocal effects originated in the depletion of the pump pulse as well as of its pedestal. In addition, it can provide amplification to the pump wave with a slight modification of the setup. Furthermore, this method can be combined with pump pulse coding and a new technique for coding linearization that we have devised to further extend the sensing length into the hundreds of kilometers range.
\end{abstract}

Keywords: stimulated Brillouin scattering, Brillouin optical time-domain analysis, non-local effects, pulse coding

\section{INTRODUCTION}

Research on Brillouin distributed sensors (BDS) has been extensive during the last three decades due to the ability of this technology to measure the distribution of temperature and strain along optical fibers, providing thousands of measurement locations along a structure. The main market for this technology is currently the oil and gas industry, where it has been deployed for more than a decade to assess the integrity of oil and gas pipelines with the detection of leaks as well as geohazard threats such as erosion, landslide and subsidence. ${ }^{1}$ These sensors have been also applied to measure temperature and strain along the umbilical cables used for subsea wells and for borehole measurements. ${ }^{2}$ The other main application area has been the monitoring of the electric grid, were BDS have been deployed for ampacity and thermal rating of high voltage cables, particularly in underground or subsea installation. ${ }^{3}$ There are other fields where the application of the BDS technology is being explored at the moment such in civil, geotechnical or aerospace engineering. However, much research is still needed to achieve significant deployments of the technology in these and other fields were the engineering involved in developing the application is significant.

We are concerned here with BDS of the Brillouin optical time-domain analysis (BOTDA) type, which provide the best performance for the long-range measurements applications which are the main market drivers for the technology. These sensors are based on the use of a high power pump pulse that propagates along the fiber and interacts with a counter-propagating probe wave at each position via stimulated Brillouin scattering. The performance of a BOTDA is determined by the magnitude of this interaction and the intensity change that it induces on the detected probe wave. The larger the pump pulse power at a given position, the greater the gain or loss (depending on sensor configuration) induced on the probe wave wavefront. Moreover, the greater the probe power the bigger the total intensity step finally detected due to that gain or loss. The main parameters that quantify the final performance of a BOTDA setup are the spatial resolution, the distance range, the measurement time and the measurement precision. All of these parameters are interdependent.

The most fundamental restriction to the performance of BOTDA sensors in the attenuation of the optical fiber. Its deleterious effect is twofold: first, it attenuates the pump wave as it travels along the fiber so that the gain induced upon the probe wave at a particular location is reduced. And second, it attenuates the probe wave as it propagates to the receiver so that the induced gain translates to smaller intensity change at the receiver and hence lower performance. In principle, this reduction in Brillouin interaction between the pump pulse and probe

Further author information: (Send correspondence to A.L.)

A.L.: E-mail: alayn.loayssa@unavarra.es 
wave could be compensated by increasing the pump and probe powers. However, these powers are constrained by several effects. The pulse power is constrained by the onset of nonlinear effects such as modulation instability, self-phase modulation and, finally, Raman scattering. ${ }^{4,5}$ The magnitude of these effects can be reduced by various methods, but they ultimately set a limit on the maximum power that can be deployed, which is in the 100-mW range for typical fiber parameters. As for the probe wave, its power is first limited by the onset of so-called nonlocal effects. These are due to the transfer of energy from the pump pulse to the probe wave during their interaction along the sensing fiber. This transfer has been shown to introduce an additional transfer function in the measurement due to optical frequency-dependent pump depletion, which induces a systematic error in the measurement of the Brillouin frequency shift (BFS) ${ }^{6,7}$ A second higher-power limit for the probe power that can be injected in the sensing fiber link is the Brillouin threshold of the fiber, over which significant noise is added to the detected probe signal due to spontaneous Brillouin scattering. ${ }^{8}$

In this work, we review several techniques that have been recently proposed to enhance the performance of long-range BOTDA sensors. We focus on our contributions based on the application of the so-called probe dithering method. This is a simple but powerful technique that is based on introducing a wavelength modulation to the probe wave in a conventional BOTDA setup. We show that this technique can be used to compensate nonlocal effects originated in the depletion of the pump pulse or of its pedestal so that the probe wave power can be taken to the Brillouin threshold of the sensing fiber, which itself is increased by the deployment of probe dithering. Furthermore, a slight modification of the probe-dithered BOTDA setup leads to the introduction of distributed gain on the pump pulse that serves to compensate fiber attenuation. Finally, we show that probe dithering can be combined with pump pulse coding and with a new technique for coding linearization that we have devised to further extend the sensing length.

The paper is organized as follows. First, in section 2, the fundamentals of the probe dithering technique are introduced. Then, section 3 describes how this technique can be used to compensate nonlocal effects originated in the depletion of the pump pulse. This is followed by section 4, in which we also demonstrate that probe dithering can be used to mitigate nonlocal effects originated in the depletion of the pedestal of the pulse. Finally, section 5 summarizes our contributions combining probe dithering with pump pulse coding.

\section{FUNDAMENTALS OF THE PROBE DITHERING TECHNIQUE}

We start by outlining the fundamentals of the probe dithering technique, which is the fundamental tool that we have been using to enhance the performance of long-range BOTDA sensors. The basis of this technique is to introduce a modulation or "dithering" to the optical frequency (wavelength) of the probe wave, as it is schematically depicted in Fig. 1, where the optical waves involved in the technique as well as its results on the Brillouin interaction between pump and probe waves are highlighted. As is depicted in the figure, the optical frequency of the probe wave is modulated in the time domain with a given function that can be sinusoidal, triangular, sawtooth, etc. In addition, this frequency modulation (FM) of the probe wave is synchronized to the launching of the pump pulses into the fiber, so that a series of pulses experience the same optical frequency of the probe waves at any given location. This makes the effective optical frequency of the probe wave meeting the pump pulses at each location to vary along the optical fiber following the applied FM modulation shape. The resulting Brillouin interaction between both waves depends on $\Delta \nu$, the detuning of the interaction from the center of the Brillouin spectrum at each position of the fiber: ${ }^{9}$

$$
\Delta \nu(z)=\nu_{P}-\nu_{S}(z)-B F S(z)
$$

where $\nu_{P}$ is the pump pulse wavelength, $\nu_{S}$ is the probe wave wavelength at position $z$ and BFS is the Brillouin frequency shift. Note that the last expression points out that it is equivalent to have an actual variation in BFS due to the fiber characteristics than to have that same variation in $\nu_{S}$. Therefore, a virtual BFS profile is synthesized by the probe wavelength modulation. The net effect is completely analogous to having a virtual BFS distribution in the fiber, in the sense of having a fiber whose BFS profile has the same shape as the FM modulation imposed upon the probe.

With the probe dithering technique, there are two possibilities to scan the Brillouin spectra by sweeping $\Delta \nu$ at all locations in the fiber. The first one is to sweep the difference between the optical frequency of the pump 
and probe waves, $\nu_{P}-\nu_{S 0}$, where $\nu_{S 0}$ is the mean frequency value of the FM modulated probe wave. This provides experimental measurements of probe gain that can be fitted to the Brillouin gain profile to obtain the virtual BFS profile. The real BFS is then obtained by subtracting the known probe FM modulation for the measured virtual BFS. The disadvantage of using this method is that a measurement time penalty is incurred because the probe wavelength modulation increases the span of optical frequencies that need to be swept, as it is equivalent to increasing the variation of the BFS profile along the fiber. The measurement time penalty factor is given by:

$$
T=\frac{2 \Delta f_{p}+\Delta f_{T}}{\Delta f_{T}}
$$

where $\Delta f_{p}$ is the peak frequency deviation of the FM modulation of the probe wave and $\Delta f_{T}$ is the frequency range that would be swept in the conventional BOTDA without probe dithering. However, for realistic scenarios, this penalty can be rather small. For instance, if we assume a typical long-range measurement with $\Delta f_{T}=1 \mathrm{GHz}$, which can be quite realistic in a long-range link made of several fiber types, and $\Delta f_{p}=100 \mathrm{MHz}$, as it is deployed in the experiments below, then the penalty would be just a $20 \%$ increment in the measurement time. The other alternative to scan the Brillouin spectra along the fiber when deploying probe dithering is by sequentially changing the relative delay between the pump pulses and the FM wave, as it is schematically depicted in Fig. 1. This changes the relative location of the optical frequency profile of the probe wave along the fiber so that all frequencies detunings are sequentially scanned for all measurements locations. The advantage of this method is that there is no penalty whatsoever in the measurement time compared to a conventional BOTDA.

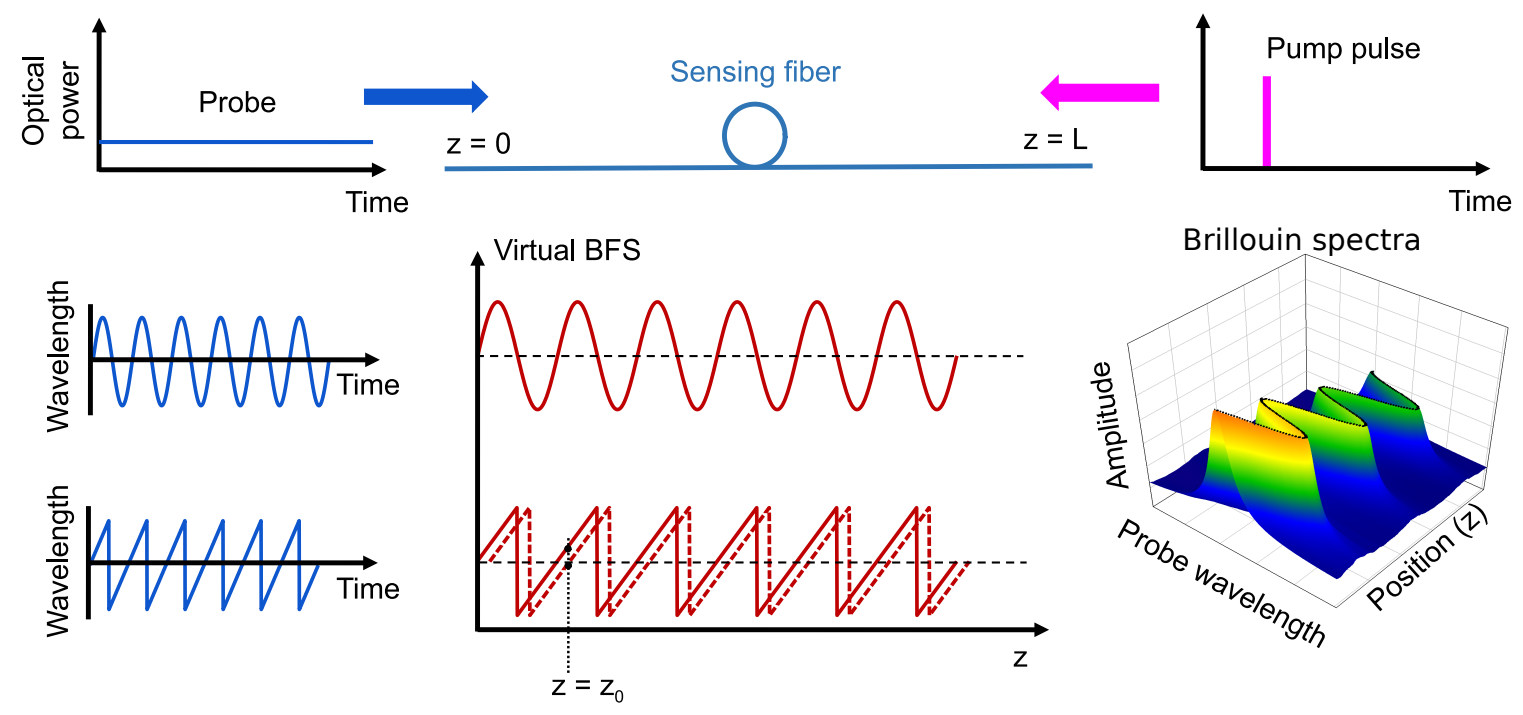

Figure 1. Fundamentals of probe dithering. The probe wave is FM modulated so that a virtual BFS profile is created along the fiber. Examples showing sinusoidal and sawtooth modulation are depicted. For the sawtooth, the method to scan different frequencies of the Brillouin by delaying the modulation is highlighted. For the sinusoidal modulation, the resultant distribution of Brillouin gain spectra along the fiber is displayed.

Regarding the experimental implementation of the probe dithering method, several configurations are possible. For instance, it can be deployed by taking advantage of the chirp of the laser used as a common source for probe and pump waves. ${ }^{9}$ Another option, which can be used in experimental setups in which the probe wave is obtained as a modulation sideband of the laser source, is to generate the FM modulation of the probe by using an RF FM wave to generate the sideband. ${ }^{10}$

\section{COMPENSATION OF NONLOCAL EFFECTS DUE TO PUMP PULSE DEPLETION}

When the pump and probe waves deployed in BOTDA sensors have an optical frequency detuning close to the BFS of the fiber, they interact exchanging energy via stimulated Brillouin scattering (SBS). In a gain-based BOTDA, 
a pump pulse at higher optical frequency transfers energy to the probe wave, which undergoes amplification. It is this localized amplification of the probe what is used for sensing. As a side-effect of this interaction, the pump waves becomes depleted as it travels along the fiber meeting successive wavefronts of the probe wave. This depletion increases with the power of the probe wave and with the length of the fiber. Moreover, the depletion of the pump wave translates into a distortion of the measured Brillouin spectrum because the gain experienced by the probe wave depends on the pump power. The worst-case scenario takes place when a long fiber length with uniform BFS is followed by a small section with different BFS, where the gain spectrum is measured. The pump power arriving at the end of the fiber experiences a depletion that depends on the frequency detuning between pump and probe optical waves along the long fiber length. This frequency dependence introduces an additional transfer function being superimposed on the measured gain spectrum at the small section. This distorts the measured spectrum and introduces a bias in the obtained BFS that entails a systematic error in the measurement performed. ${ }^{7}$ Notice that this measurement impairment is a nonlocal effect in the sense that the BFS measurement at a particular location in the fiber is affected by the Brillouin interaction between pump and probe at other locations. For typical long-length standard single-mode fiber sensing link, this effect translates into an upper limit of $-14 \mathrm{dBm}$ on the maximum probe power that can be deployed. ${ }^{7}$

The pump pulse depletion issue has been known since the beginnings of the development of BOTDA sensors. Therefore, several methods have also been introduced over the years to mitigate this nonlocal effects such as the use of post-processing for BFS profile reconstruction, ${ }^{11}$ or a time-multiplexing method that is based on pulsing the probe wave so as to limit the interaction length with the pump pulse. ${ }^{12,13}$ However, these are complex methods that increase the measurement time and are not suitable for use in realistic application scenarios. We have also introduced a BOTDA setup, which is based on deploying a phase-modulated probe wave and RF demodulation, that provides tolerance to pump pulse depletion nonlocal effects. ${ }^{14}$

Nevertheless, the simplest and the most successful alternative to mitigate nonlocal effects due to pump depletion is the use of the so-called dual-probe setup, which uses two probe waves, equally and symmetrically separated in optical frequency from the pump. ${ }^{15}$ These induce two simultaneous Brillouin interactions (gain and loss) upon the pulse that compensate each other; hence, avoiding depletion. However, this is only true to first order approximation neglecting the amplification and attenuation experienced by the lower and higher frequency probes, respectively, during their interaction with the pump. When the detailed interaction is taken into account, it is found that in a dual-probe BOTDA there is still pump depletion, although it is greatly diminished. ${ }^{7}$ Furthermore, later work showed that the probe power limitation in this setup was actually more restrictive due to the onset of second-order nonlocal effects. These nonlocal effects are related to the appearance of linear distortion of the pump pulse spectrum due to its interaction with the two probe waves as the frequency detuning between the pump and probe waves is scanned to retrieve the full Brillouin gain spectra. ${ }^{16}$ The simultaneous Brillouin gain and loss interactions induced by both probe waves upon the pulse do not overlap perfectly in frequency, leading to linear distortion of the pump pulse spectrum, which in turn leads to a distortion of the measured gain spectra and a BFS measurement error. ${ }^{10}$ Altogether, these effects constrain the maximum probe wave power that can be deployed in dual-probe BOTDA setups to around $-3 \mathrm{dBm}$ for typical standard single-mode fiber parameters.

One approach to overcome the distortion of the pump pulse spectrum is the use of an alternative scanning method that changes the conventional scanning of the Brillouin spectra to ensure the continuous overlapping of the gain and loss spectra induced by both probe waves on the pump ${ }^{17} .{ }^{18}$ These methods enable an increase of the probe power over the second-order nonlocal effects limit, at the cost of a somewhat increased complexity of the setup. Nevertheless, the maximum probe wave power injected into the fiber is still limited by its Brillouin threshold, which is around $7 \mathrm{dBm}$ for typical single-mode fiber.

Our approach to compensate impairments associated with the depletion of the pump pulse has been to deploy our probe dithering technique as schematically depicted in Fig. 2. A standard dual-probe BOTDA setup is deployed but the optical frequency of both probe waves is modulated in the time domain following a saw-tooth shape. This makes the Brillouin interaction induced by both probe waves upon the optical pulse to spread over a frequency range approximately equal to the peak-to-peak frequency deviation of the FM modulation. Therefore, the accumulated gain and loss spectra induced by both probe waves in their interaction with the pump pulse along the fiber broadens and flattens so that no linear distortion is introduced in the pump pulse spectrum and 
second-order nonlocal effects are suppressed. ${ }^{10}$ In addition, the gain and loss spectra mutually compensate, so there is no depletion of the pulse.

A side benefit of the probe dithering method is that the optical frequency modulation of the probe waves allows overcoming the Brillouin threshold limit of the fiber, by means of reducing the effective amplification of spontaneous Brillouin scattering. ${ }^{9}$ Therefore, a very large probe power can be deployed to enhance the signalto-noise ratio (SNR) in detection, which in turn leads to an enhancement of the measurement precision and/or the measurement time of the BOTDA sensor.

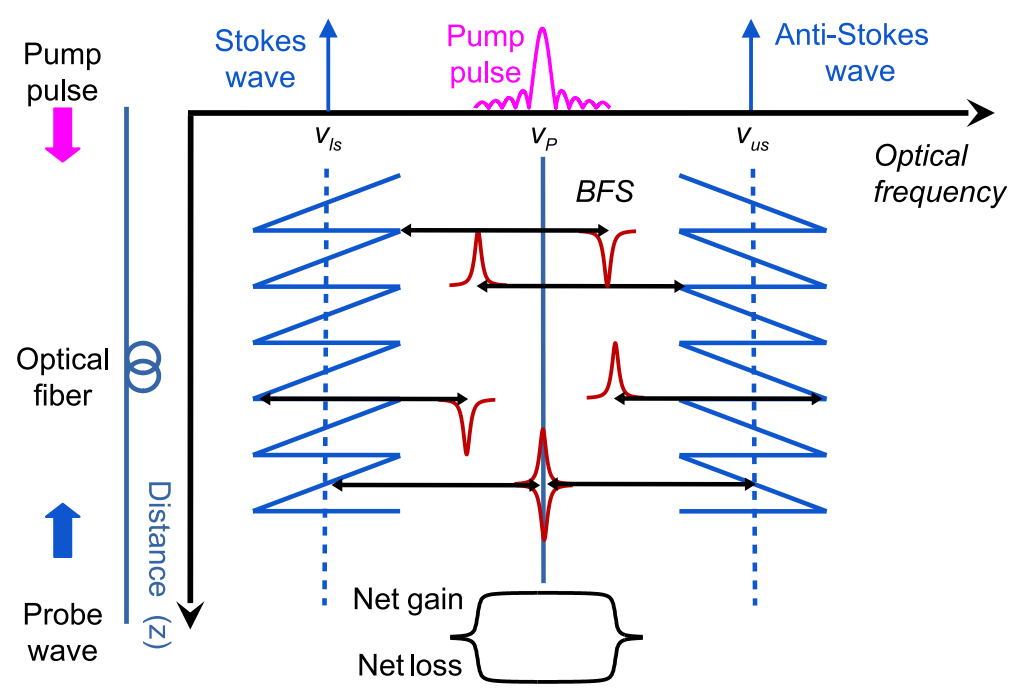

Figure 2. Fundamentals of the probe dithering technique for mitigation of second-order nonlocal effects.

The ideal and full compensation of pump pulse depletion effects via probe dithering requires that the BFS of the fiber remains uniform along the whole sensing link, although the method has been shown to be quite resilient to application in sensing links in which a single fiber type is deployed and BFS variations take place just in response to local alteration of the environmental conditions (temperature or strain). However, this is not a realistic scenario in many practical applications because in a long-range sensing link there may be fiber from several manufacturers with slightly different nominal BFSs. In order to let the technique operate correctly in such situations, we have devised a method that introduces an additional wavelength modulation to the probe wave so as to track the BFS changes along the sensing fiber link. ${ }^{19}$ This has allowed the probe dithering technique to be demonstrated in a 120-km fiber sensing link deploying three types of single-mode fibers with different nominal BFS. The use of a $15-\mathrm{dBm}$ probe wave power provided $3-\mathrm{m}$ resolution and $2-\mathrm{MHz}$ precision BFS measurement in the worst-contrast position.

Finally, the probe dithering technique can be slightly modified to provide an additional enhancement to the performance of BOTDA sensors in the form of amplification of the pump pulses as they propagate along the fiber. This is highly desirable because the attenuation of the fiber makes the pulse power arriving at the furthest end of the sensing fiber very small, which translates in small gain to the probe gain and hence reduced precision in the determination of BFS from those locations. A hint on how to achieve this pulse gain can be obtained from observation of Fig. 2. Notice that if the lower optical frequency probe is removed, the pump pulse experiences the gain induced by the higher frequency probe. Moreover, as this gain is broad and flat, all frequency components of the pulse are equally amplified, leading to a non distorted pulse, free from second-order nonlocal effects. We have experimentally demonstrated this idea in a 100-km BOTDA sensor, obtaining a measurement uncertainty of 1 $\mathrm{MHz}$ at the worst-contrast position for 1-m spatial resolution. ${ }^{20}$ Fig. 3 depicts the Brillouin spectra distribution measured along the fiber for this system. Notice that the amplitude of the measured spectra is reduced as the pulse propagates along the fiber. However, by mid-span, the amplitude starts to recover when the gain provided by the probe wave starts to become significant. 


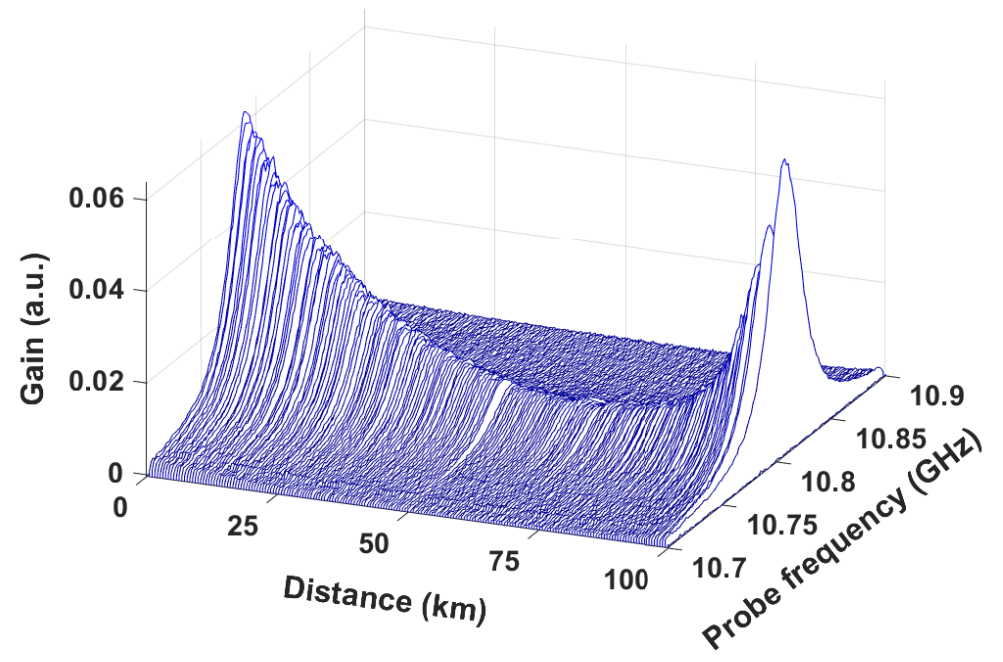

Figure 3. Brillouin spectra distribution measured in a system deploying probe dithering configured for distributed pump pulse amplification.

\section{MITIGATION OF NONLOCAL EFFECTS DUE TO THE PUMP PULSE PEDESTAL}

Another significant constrain for BOTDA sensors comes from the limited extinction ratio (ER) of the deployed pump pulses, which means that the pump pulse is on top of a pedestal. In fact, we have recently discovered two ER-related nonlocal effects that can seriously impair the performance of BOTDA sensors. ${ }^{21}$ The first impairment is due to the increased depletion of the part of the pedestal following the pump pulse (trailing pedestal) by probe wavefronts amplified by the pulse itself. This effect has been shown to introduce a distortion in the measured Brillouin spectrum that is similar to that induced by the depletion of the pulse by the probe wave. ${ }^{21}$ The other impairment is due to the interplay between the pulse pedestal and the transient response of the erbium-doped fiber amplifiers (EDFA) that are normally deployed to amplify the pump wave before injection in the sensing fiber. The transient response of these devices also distorts the trailing pedestal of the pulse inducing another type of nonlocal effect. ${ }^{21}$ The net result of both effects is an error in the determination of the BFS along the fiber that increases for smaller ER of the pump pulse. ${ }^{21}$

These ER-related nonlocal effects become significant when optical devices with medium ER are used to shape the pump pulses, for instance, the ubiquitous Mach-Zehnder electrooptic modulators (MZ-EOM). These are broadband and cost-effective devices that are suitable to provide the sharp rise and fall times that are required by BOTDA sensors with sub-meter resolution. High-ER devices such as semiconductor optical amplifiers are not able to provide the sharp transitions required in this case.

We have demonstrated the application of the probe dithering method to reduce the impairments induced by the ER of the pump pulses. In this case, dithering is applied to both waves, the probe and the pump wave including the pump pulse and the pump pedestal. The principle, which is schematically depicted in Fig. 4, is similar to that applied in Brillouin optical coherence-domain analysis (BOCDA) sensors to localize measurements. The pump pedestal and the probe wave are modulated by the same sinusoidal function. Then, the frequency difference between the pump pedestal and probe becomes a sinusoidal function of distance along the fiber that behaves like a standing wave; there are nodes (correlation peaks) where the frequency difference between both waves is constant. The Brillouin interaction between the pump pedestal and the probe at these correlation peaks is highly efficient, whereas it becomes inefficient outside these locations. The net effect is that the total Brillouin interaction between the pump pulse pedestal and the probe wave is reduced, as it is confined mainly to the correlation peaks. 

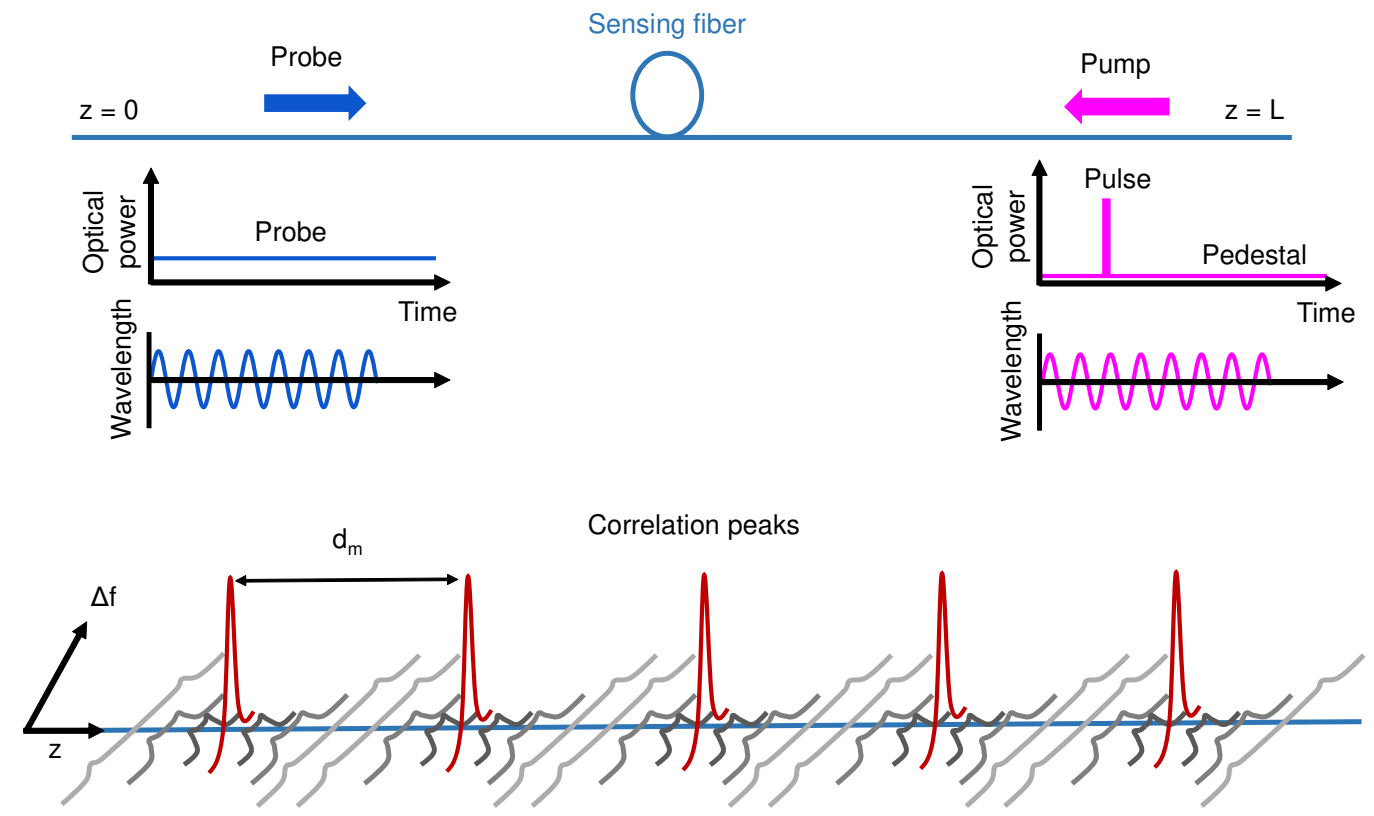

Figure 4. Fundamentals of the use of probe dithering to mitigate nonlocal effects associated with the limited extinction ratio of the pump pulses.

We have demonstrated this method in proof-of-concept experiments in a conventional 25-km BOTDA setup. In this system, the simultaneous FM modulation of the probe wave and the pump pedestal with the same signal is obtained by taking advantage of the chirp of the laser diode used to generate both waves when direct sinusoidal modulation of its current is used. Fig. 5 highlights how the use of probe dithering removes the distortion observed in the probe gain traces. With dithering, the traces depict "spikes" due to the probe FM, but the important fact is that the maxima of this spikes, which coincide with the maxima of the Brillouin spectra, coincide with the gain for the measurements for high ER pulses, hence demonstrating that the gain distortion introduced by ER-originated NLE is compensated.

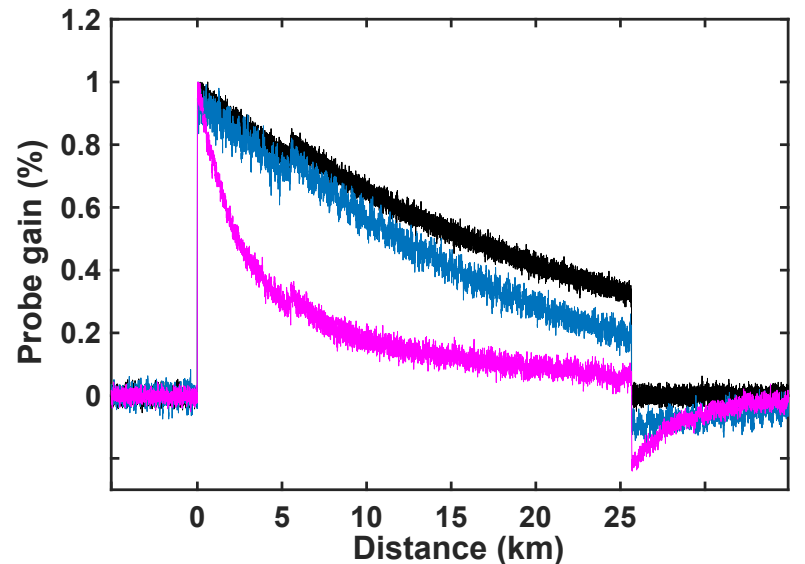

(a)

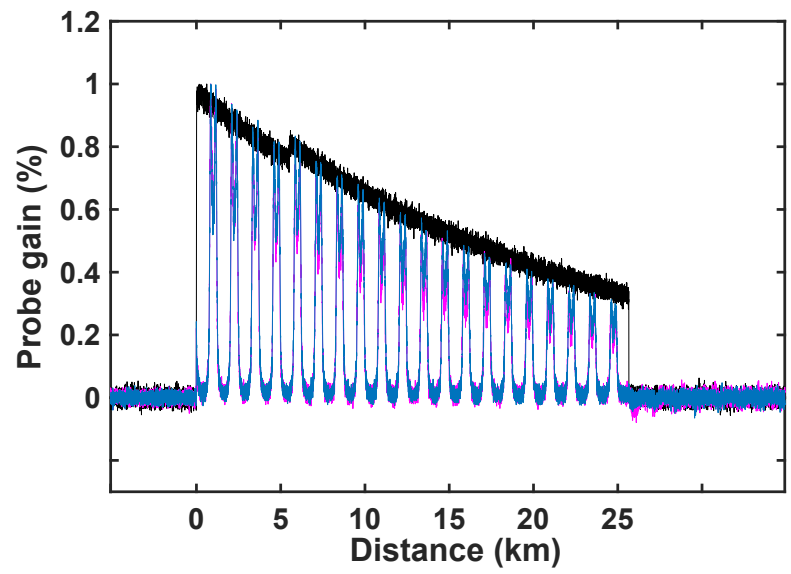

(b)

Figure 5. Probe gain profile measured along the fiber for different ER values of the pump pulse and using two EDFA models: (a) without dithering of the light source and (b) when the dithering is turned on. ER=45dB and EDFA I (black), $\mathrm{ER}=26 \mathrm{~dB}$ and EDFA I (blue), ER=26dB and EDFA II (pink). 


\section{PULSE CODING WITH PROBE DITHERING}

As it has been explained in the introduction, the range of BOTDA sensors is ultimately constrained by the attenuation of the optical fiber used for sensing because the maximum pump and probe powers that can be deployed are limited by the onset of nonlinear and nonlocal effects. Multiple solutions have been proposed to increase the range of BOTDA sensors, but one of the most effective is the deployment of pump pulse coding. ${ }^{22}$ These methods are based on launching in the fiber coded sequences of pulses and processing the detected probe signals so as to obtain, after a decoding process, a probe intensity response equivalent to that of the single-pulse BOTDA, but with an enhancement in SNR, known as coding gain, that increases with the code length. This enhancement is due to the increased pump energy in the fiber and it does not compromise the spatial resolution of the sensor. Coding techniques were first applied to optical time-domain reflectometers and Raman distributed temperature sensors, but, eventually, they found their way to BOTDA sensors. ${ }^{22}$ The most useful codes are variations of the Simplex ${ }^{23}$ and complementary-correlation Golay codes. ${ }^{24}$

Pump pulse conding can provide a significant enhancement to the performance of BOTDA sensors, but their application is constrained by three factors. First, the application of coding techniques requires that, in order to properly decode the signal, all pump pulses in the coded sequence have the same power. This is complicated by the fact that the EDFAs that are typically deployed to amplify the pulses before injection in the fiber have a transient response that distorts the amplitude of a burst of pulses. However, methods to mitigate the effects of this transient response have been demonstrated by using specialty EDFA for amplification of bursts of pulses or by using schemes that introduce additional pulses in the EDFA to flatten its response. ${ }^{25}$

The second limitation to the deployment of pump pulse coding methods comes from a worsening of nonlocal effects related to pump depletion when multiple pump pulses are launched simultaneously into the sensing fiber. Successive pulses in the coded sequence interact with probe wavefronts that have been already amplified by previous pulses. Therefore, the amount of pump depletion experienced by the single-pulses, instead of being just related to the power of the probe wave injected in the fiber, also depends on the amplification of the probe wavefronts by previous pulses in the coded sequence. This means that the independence of pump pulse depletion on pump pulse power that applies to single-pulse BOTDA sensors does no longer hold. In a BOTDA system with coding, the higher the pump pulse power, the larger the amplification of the probe wave by previous pulses in a sequence, and hence, the larger the depletion of a particular pump pulse. Hence, nonlocal effects will appear earlier in BOTDA setups using pump pulse coding than in conventional single-pulse BOTDA sensors. Furthermore, even for a dual-probe wave BOTDA configuration, which mitigates nonlocal effects, the presence of multiple pulses in the fiber when using coding makes pump depletion to become significant at lower input probe wave power than for single-pulse dual-probe systems. Therefore, nonlocal effects in BOTDA sensors with coding constrain the probe power as well as the pump pulse power compared to the sensor without coding, which is counterproductive for the sensor performance.

Nonlocal effects related to pump depletion in pulse-coded BOTDA sensors manifest as a distortion in the decoded probe gain traces that lead to errors in the determination of BFS. As explained before, each pulse in a coded sequence experiences a differential depletion along the fiber. Therefore, the effect is similar to that of the transient response of the EDFA in the sense that the pump pulses in the coded sequence no longer have the same power, which leads to failure of the decoding process.

The final constrain on the use of pump pulse coding is the requirement for linearity of the decoding process. In order for decoding to work, it is necessary that contributions of individual pulses to the detected signal combine linearly. However, that is not exactly the case for BOTDA sensors because the gain of the probe wave depends on the exponential of the pump pulse power. In pulse-coded BOTDA, the total intensity variation experienced by the probe when interacting with particular codeword sequences of pulses in the fiber, $\Delta I_{C W_{T}}$, is given as:

$$
\Delta I_{C W_{T}}(t)=I_{C W_{L}} \exp (-\alpha L)\left[\exp \left(\sum_{i=1}^{L_{C}} g_{i}\left(z_{i}\right)\right)-1\right] \approx I_{C W_{L}} \exp (-\alpha L) \sum_{i=1}^{L_{C}} g_{i}\left(z_{i}\right),
$$

where $I_{C W_{L}}$ is the probe power injected in the fiber of length $L$ and attenuation coefficient $\alpha$, and $g_{i}$ is the gain experienced by the probe due to its interaction with the $i$-th pulse in the codeword sequence at a location 
$z_{i}$ along the fiber. Notice that the last approximation on the right-hand side of (4) is required to comply with the linearity of the coding process. However, this approximation is just valid when $\sum_{i=1}^{L_{C}} g_{i}\left(z_{i}\right)$ is small so that it becomes unreasonable as the code length, and hence the total gain, increases. This makes the recovery of $g(z)$, the single-pulse gain, from the measured $\Delta I_{C W_{T}}$ for each codeword to fail, because the decoding process intrinsically requires that the effects of multiple pulses accumulate linearly. Altogether, this linearity requirement constrains the maximum code length that can be deployed in BOTDA sensors and, with it, the maximum achievable SNR enhancement and overall measurement range.

Several solutions have been proposed to mitigate the constrains related to nonlocal effects and the linearity requirement in pump pulse coded BOTDA sensors. One alternative is the use of color coding, which is based on introducing an additional optical frequency hopping to the pulse sequence. ${ }^{26}$ This additional and continuous frequency change of the pump pulses of a given sequence reduces the total gain experienced by the probe because only part of the pump pulses in a codeword are close to the Brillouin gain peak and lead to significant interaction. Another proposal is the use of bipolar coding, which is based on using codes that include pulses that are upshifted or downshifted in optical frequency from the probe so that they induce either gain or loss on the probe wavefront. ${ }^{27}$ This ensures that the total gain or loss experienced by the probe after transversing the fiber is small; hence, linear conditions prevail. These approaches have demonstrated good performance at the cost of a rather increased setup complexity.

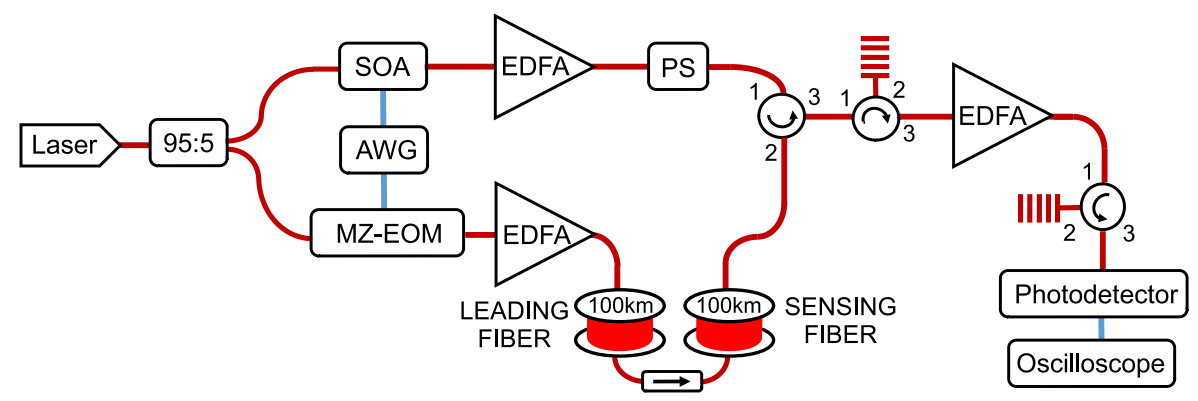

Figure 6. Experimental setup for application of probe dithering and linearization in a pulse-coded BOTDA sensor.

We have demonstrated the use of probe dithering to compensate nonlocal effects in pulse coding BOTDA. ${ }^{28}$ The application of the method is straightforward. The only additional requirement compared to the application in single-pulse BOTDA is the need to use a code period that is equal or a multiple of the period of the FM modulation of the probe deployed in order for the decoding process to work properly. This ensures that, at a given location in the fiber, successive pulses in a sequence meet the probe wave with the same frequency detuning.

In addition, we have devised a very simple technique to ensure the linearity of pulse coding in BOTDA. ${ }^{29} \mathrm{It}$ is based in using a linearized version of the intensity variation that is calculated by taking the natural logarithm of the detected probe intensity wave and subtracting its DC component:

$$
\begin{aligned}
\Delta I_{C W_{T}}^{\prime}(t) & =\ln \left(I_{C W_{L}} \exp (-\alpha L) \exp \left(\sum_{i=1}^{L_{C}} g_{i}\left(z_{i}\right)\right)\right)-\ln \left(I_{C W_{L}} \exp (-\alpha L)\right) \\
& =\sum_{i=1}^{L_{C}} g_{i}\left(z_{i}\right)=\sum_{i=1}^{L_{C}} \Delta I_{C W_{i}}^{\prime}(t)
\end{aligned}
$$

where $\Delta I_{C W_{i}}^{\prime}(t)$ is the linearized probe intensity variation due to interaction of the probe wave with the $i$-th pulse in the codeword. Apart from this really simple modification, the measurement process then proceeds identically to established procedures. First, $I_{C W_{T}}(t)$ is detected for the different codewords and $\Delta I_{C W_{T}}^{\prime}(t)$ calculated for each of them, according to (4). Then, decoding is performed.

We applied both methods, probe dithering, and coding linearization, to a BOTDA setup with a 200-km fiber loop. The experimental setup is depicted in Fig. 6. This is a conventional dual-probe BOTDA setup in which 


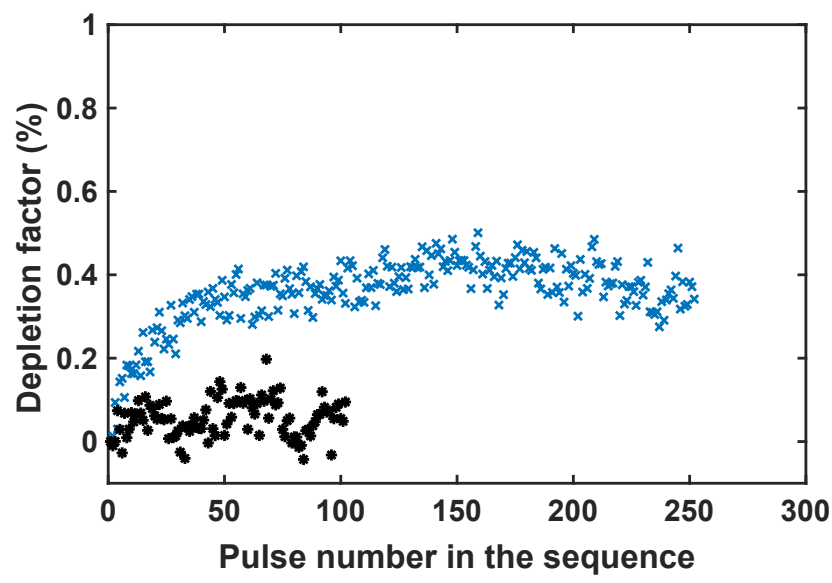

(a)

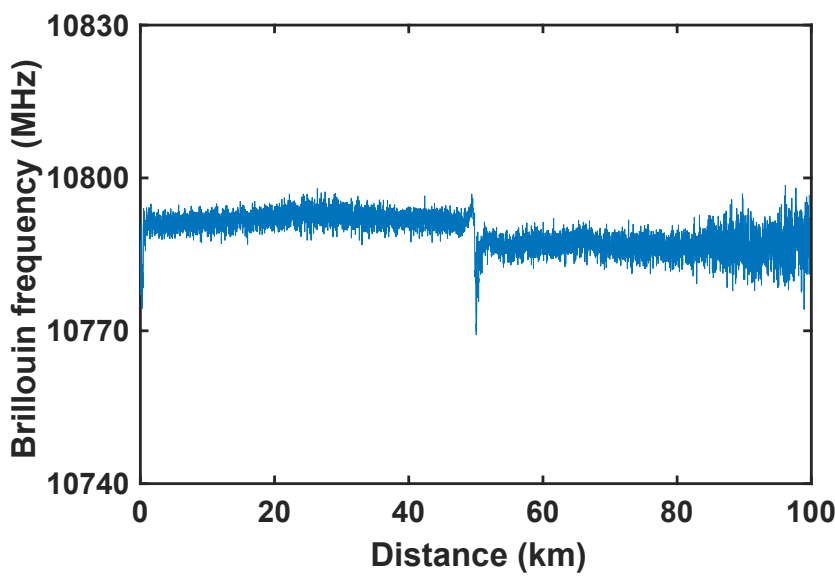

(b)

Figure 7. Experimental results of a 200-km BOTDA sensor deploying probe dithering and coding linearization. (a) Depletion factor of the pulses in coded sequences for $L_{c}=103$ (black) $L_{c}=251$ (blue). (b) BFS distribution along the fiber for $L_{c}=251$.

and arbitrary waveform generator is used to generate an FM modulation of the probe wave following a sawtooth shape with $160-\mathrm{MHz}$ peak-to-peak frequency deviation and a period of $4 \mu \mathrm{s}$. This modulation made it possible to inject a total of $13 \mathrm{dBm}$ of probe power into the sensing link. An optimized cyclic simplex code ${ }^{30}$ was with a code length of up to $L_{c}=251$. Fig. 7 (a) depicts the deplection factor of the pulses in the sequence, where it can be seen that deployment of the probe dithering made the pump depletion negligible. Finally, Fig. 7 (b) depicts the measurement of the BFS along the sensing fiber. The measurement precision was better that $2 \mathrm{MHz}$ with a spatial resolution of $2 \mathrm{~m}$.

\section{CONCLUSIONS}

In summary, we have introduced the application of the probe dithering method to enhance the performance of long-range BOTDA sensors. It has been demonstrated that this technique is rather efficient in compensating the deleterious effects of nonlocal effects and that it can lead to significant enhancements in performance when combined with pump pulse coding methods, particularly if our technique for coding linearization is also deployed. Moreover, the technique requires minimal alteration of the conventional BOTDA setups. Therefore, its use in realistic application scenarios is straightforward and cost effective.

\section{ACKNOWLEDGMENTS}

The authors wish to acknowledge the financial support of the Spanish Agencia Estatal de Investigacin through projects TEC2016- 76021-C2-1-R and RTC-2017-6239-3, FEDER funds, Gobierno de Navarra project 0011-13652017-000122 and the Universidad Pública de Navarra.

\section{REFERENCES}

[1] Madabhushi, S. S. C., Elshafie, M. Z. E. B., and Haigh, S. K., "Accuracy of distributed optical fiber temperature sensing for use in leak detection of subsea pipelines," Journal of Pipeline Systems Engineering and Practice 6(2), 04014014 (2015).

[2] Hartog, A. H., [An introduction to distributed optical fibre sensors], CRC Press (2017).

[3] Ukil, A., Braendle, H., and Krippner, P., "Distributed temperature sensing: Review of technology and applications," IEEE Sensors Journal 12, 885-892 (May 2012).

[4] Stella M. Foaleng, L. T., "Impact of Raman scattering and modulation instability on the performances of Brillouin sensors," Proc.SPIE 7753, 7753 - 7753 - 4 (2011). 
[5] Foaleng, S. M., Rodríguez-Barrios, F., Martin-Lopez, S., González-Herráez, M., and Thévenaz, L., "Detrimental effect of self-phase modulation on the performance of Brillouin distributed fiber sensors," Opt. Lett. 36, 97-99 (Jan 2011).

[6] Geinitz, E., Jetschke, S., Rpke, U., Schrter, S., Willsch, R., and Bartelt, H., "The influence of pulse amplification on distributed fibre-optic Brillouin sensing and a method to compensate for systematic errors," Measurement Science and Technology 10(2), 112 (1999).

[7] Thévenaz, L., Mafang, S., and Lin, J., "Effect of pulse depletion in a Brillouin optical time-domain analysis system," Optics Express 21(12), 14017-14035 (2013).

[8] Shimizu, T., Nakajima, K., Shiraki, K., Ieda, K., and Sankawa, I., "Evaluation methods and requirements for the stimulated Brillouin scattering threshold in a single-mode fiber," Opt. Fiber Tech. 14(1), 10 - 15 (2008).

[9] Urricelqui, J., Sagues, M., and Loayssa, A., "Synthesis of Brillouin frequency shift profiles to compensate non-local effects and brillouin induced noise in BOTDA sensors," Opt. Express 22, 18195-18202 (Jul 2014).

[10] Ruiz-Lombera, R., Urricelqui, J., Sagues, M., Mirapeix, J., López-Higuera, J. M., and Loayssa, A., "Overcoming nonlocal effects and Brillouin threshold limitations in Brillouin optical time-domain sensors," IEEE Photonics Journal 7, 1-9 (Dec 2015).

[11] Minardo, A., Bernini, R., Zeni, L., Thevenaz, L., and Briffod, F., "A reconstruction technique for long-range stimulated Brillouin scattering distributed fibre-optic sensors: experimental results," Measurement Science and Technology 16(4), 900 (2005).

[12] Dong, Y., Chen, L., and Bao, X., "Time-division multiplexing-based BOTDA over 100km sensing length," Opt. Lett. 36, 277-279 (Jan 2011).

[13] Zornoza, A., Minardo, A., Bernini, R., Loayssa, A., and Zeni, L., "Pulsing the probe wave to reduce nonlocal effects in Brillouin optical time-domain analysis (botda) sensors," IEEE Sensors Journal 11, 1067-1068 (April 2011).

[14] Urricelqui, J., Sagues, M., and Loayssa, A., "BOTDA measurements tolerant to non-local effects by using a phase-modulated probe wave and rf demodulation," Opt. Express 21, 17186-17194 (Jul 2013).

[15] Minardo, A., Bernini, R., and Zeni, L., "A simple technique for reducing pump depletion in long-range distributed Brillouin fiber sensors," IEEE Sensors Journal 9, 633-634 (June 2009).

[16] Dominguez-Lopez, A., Angulo-Vinuesa, X., Lopez-Gil, A., Martin-Lopez, S., and Gonzalez-Herraez, M., "Non-local effects in dual-probe-sideband Brillouin optical time domain analysis," Opt. Express 23, 1034110352 (Apr 2015).

[17] Dominguez-Lopez, A., Yang, Z., Soto, M. A., Angulo-Vinuesa, X., Martin-Lopez, S., Thevenaz, L., and Gonzalez-Herraez, M., "Novel scanning method for distortion-free BOTDA measurements," Optics Express 24(10), 10188-10204 (2016).

[18] Hong, X., Lin, W., Yang, Z., Wang, S., and Wu, J., "Brillouin optical time-domain analyzer based on orthogonally-polarized four-tone probe wave," Opt. Express 24, 21046-21058 (Sep 2016).

[19] Mompo, J., Iribas, H., Urricelqui, J., and Loayssa, A., "Second-Order Nonlocal Effects Mitigation in Brillouin Optical Time-Domain Analysis Sensors by Tracking the Brillouin Frequency Shift Profile of the Fiber," IEEE Photon. J. 9(5) (2017).

[20] Mompó, J. J., Urricelqui, J., and Loayssa, A., "Brillouin optical time-domain analysis sensor with pump pulse amplification," Opt. Express 24(12), 12672-12681 (2016).

[21] Iribas, H., Mariñelarena, J., Feng, C., Urricelqui, J., Schneider, T., and Loayssa, A., "Effects of pump pulse extinction ratio in Brillouin optical time-domain analysis sensors," Opt. Express 25(22), 27896-27912 (2017).

[22] Muanenda, Y. S., Taki, M., Nannipieri, T., Signorini, A., Oton, C. J., Zaidi, F., Toccafondo, I., and Pasquale, F. D., "Advanced coding techniques for long-range Raman/BOTDA distributed strain and temperature measurements," J. Lightwave Technol. 34, 342-350 (Jan 2016).

[23] Jones, M., "Using simplex codes to improve OTDR sensitivity," IEEE Photonics Technology Letters 5(7), 822-824 (1993). 
[24] Nazarathy, M., Newton, S., Giffard, R., Moberly, D., Trutna, W., Foster, S., and Sischka, F., "Real-time long range complementary correlation optical time domain reflectometer," Journal of Lightwave Technology 7(1), 24-38 (1989).

[25] Wang, F., Zhu, C., Cao, C., and Zhang, X., "Enhancing the performance of BOTDR based on the combination of FFT technique and complementary coding," Opt. Express 25, 3504-3513 (Feb 2017).

[26] Floch, S. L., Sauser, F., Llera, M., and Rochat, E., "Novel Brillouin optical time-domain analyzer for extreme sensing range using high-power flat frequency-coded pump pulses," J. Lightwave Technol. 33, 2623-2627 (Jun 2015).

[27] Soto, M. A., Floch, S. L., and Thévenaz, L., "Bipolar optical pulse coding for performance enhancement in BOTDA sensors," Opt. Express 21, 16390-16397 (Jul 2013).

[28] Iribas, H., Loayssa, A., Sauser, F., Llera, M., and Floch, S. L., "Cyclic coding for Brillouin optical timedomain analyzers using probe dithering," Opt. Express 25, 8787-8800 (Apr 2017).

[29] Mariñelarena, J., Iribas, H., and Loayssa, A., "Pulse coding linearization for Brillouin optical time-domain analysis sensors," Opt. Lett. 43, 5607-5610 (Nov 2018).

[30] Taki, M., Muanenda, Y., Oton, C. J., Nannipieri, T., Signorini, A., and Pasquale, F. D., "Cyclic pulse coding for fast BOTDA fiber sensors," Opt. Lett. 38, 2877-2880 (Aug 2013). 